\title{
Oil debris analysis and Vibration monitoring system for condition monitoring and fault diagnosis of a gearbox
}

\author{
S M Naufel Isalm', MD Farhan Mahmud Rashik², MD Khairul Basher ${ }^{3}$ \\ Student, Mechanical Engineering, China University of Mining and Technology, Xuzhou, China ${ }^{1,2,3}$
}

\begin{abstract}
Gear is the key part of the gearbox and it is important to monitor the working condition of the gear in the fault diagnosis of the gearbox. The progress and changes over the past 30 years in failure detection techniques of rotating machinery changes frequently. Oil analysis technique and vibration monitoring both are widely used in the field of fault diagnosis and condition monitoring. The purpose of this paper is to compare two systems for monitoring the condition of a gearbox.
\end{abstract}

Keywords: Oil debris analysis, vibration monitoring, gearbox condition monitoring, Fault diagnosis, Elemental Spectroscopy.

\section{INTRODUCTION}

Gearboxes often operate under fluctuating load conditions during service. Conventional techniques for monitoring vibration are based on the assumption that changes in the measured structural response are caused by deterioration in the condition of the gearbox. However, this assumption is not valid for fluctuating load conditions. It is important to detect any problems at an early stage to stay away from unexpected breakdown. In that case assumption won't work and solid detection technique is needed.

Lubricating oil is the lifeblood of the gearbox. It is required to carry out specific functions in order to keep the gearbox running. In wind turbines, the lubricant is subjected to extreme temperatures, varying load weights and contamination. Lubricant performance deteriorates under these conditions, and thus oil analysis becomes essential to monitor lubricant condition.

Another commonly used system for condition monitoring is vibration monitoring. By using some accelerometer sensor, we collect the data. When everything is working perfectly the vibration of the gearbox should be lower. If there is increasing of vibration by the rate and the peak of the graph, we can understand the cause and take necessary steps.

\section{VIBRATION MONITORING}

Vibration signal analysis has been widely used in the fault detection of rotation machinery. The vibration signal of a gearbox carries the signature of the fault in the gears, and early fault detection of the gearbox is possible by analyzing the vibration signal using different signal processing techniques.

Vibration generated by gearboxes is complicated in its structure but gives a lot of information. We may say that vibration is a signal of a gearbox condition. To understand information carried by vibration one has to be conscious/ aware of a relation between factors having influence to vibration and a vibration signal. In order to detect an impending failure, a good understanding of the evidence relating to the failure mode and methods of collecting and quantifying the evidence is needed. The common target is to detect the presence and the type of fault at an early stage of development and to monitor its evolution, in order to estimate the machine's residual life and choose an adequate plan of maintenance.

It is well known that the most important components in gear vibration spectra are the gear meshing frequency (GMF) and its harmonics, together with sidebands due to modulation phenomena. The increment in the number and amplitude of such sidebands may indicate a fault condition. Moreover, the spacing of the sidebands is related to their source. source identification and fault detection from vibration signals associated with items which involve rotational motion such as gears, rotors and shafts, rolling element bearings, journal bearings, flexible couplings, and electrical machines depend upon several factors: (i) the rotational speed of the items, (ii) the background noise and/or vibration level, (iii) the location of the monitoring transducer, (iv) the load sharing characteristics of the item, and (v) the dynamic

interaction between the item and other items in contact with it. The main causes of mechanical vibration are unbalance, misalignment, looseness and distortion, defective bearings, gearing and coupling in accuracies, critical speeds, various form of resonance, bad drive belts, reciprocating forces, aerodynamic or hydrodynamic forces, oil whirl, friction whirl, rotor/stator misalignments, bent rotor shafts, defective rotor bars, and so on. Some of the most common faults that can be detected using vibration analysis are summarized in Table1 


\section{International Advanced Research Journal in Science, Engineering and Technology}

Vol. 8, Issue 9, September 2021

DOI: $10.17148 / I A R J S E T .2021 .8922$

TABLE I

\begin{tabular}{|l|l|}
\hline \multicolumn{1}{|c|}{ Item } & \multicolumn{1}{|c|}{ Fault } \\
\hline Gears & Tooth missing faults, \\
& Misalignment \\
& Cracked and /or worm teeth \\
\hline Shaft and Rotors & Unbalance \\
& Bent shaft \\
& Misalignment \\
& Eccentric journal \\
& Loose components \\
& Rubs \\
& Critical speed \\
& Cracked shaft \\
& Blade loss \\
& Blade resonance \\
\hline Rolling element bearings & Pitting of race and ball/roller \\
& Spalling \\
& other rolling element defect \\
\hline Flexible coupling & Misalignment \\
& Unbalance \\
\hline Electrical machine & Unbalance magnetic pulls \\
& Broken /damaged roller \\
& Air gap geometric vibrations \\
& Structural foundation fault \\
& Structural resonance \\
& Piping resonance \\
\hline
\end{tabular}

\section{A. Gearbox Failure and its Vibration Analysis Techniques}

The principle causes for gear failure are given here - a) An error of design, b) An application error, c) It is likely that there is a manufacturing error.

Design errors may be due to causes like improper gear geometry, use of wrong materials, quality, lubrication and other specifications. Application errors can be due to problems like vibration, mounting and installation, cooling and maintenance while manufacturing errors can be in the form of mistakes in machining or problems in heat treating. Time domain, frequency domain, time frequency domain based on short time Fourier transform (STFT) and wavelet transform and advanced signal processing techniques have been implemented and tested.

\section{B. Time Domain Analysis}

The time domain methods try to analyze the amplitude and phase information of the vibration time signal to detect the fault of gear-rotor-bearing system. The time domain is a perceptive that feels natural, and provides physical insight into the vibration. It is particularly useful in analyzing impulsive signals from bearing and gear defects with non-steady and short transient impulses.

\section{C. $\quad$ Frequency Domain Analysis}

The frequency domain methods include Fast Fourier Transform (FFT), Hilbert Transform Method and Power Cestrum Analysis, etc. They are using the difference of power spectral density of the signal due to the fault of gear and/or bearing to identify the damage of elements. Any real-world signal can be broken down into a combination of unique sine waves. Every sine wave separated from the signal appears as a vertical line in the frequency domain. Its height represents its amplitude and its position represents the frequency. The frequency domain representation of the signal is called the signal. The frequency domain completely defines the vibration. Frequency domain analysis not only detects the faults in rotating machinery but also indicates the cause of the defect. Theoretically, time domain can be converted into frequency domain using the Fourier Transforms and vice versa. The Fourier transform is a generalization of the complex Fourier series in the limit as LLM. 
DOI: 10.17148/IARJSET.2021.8922

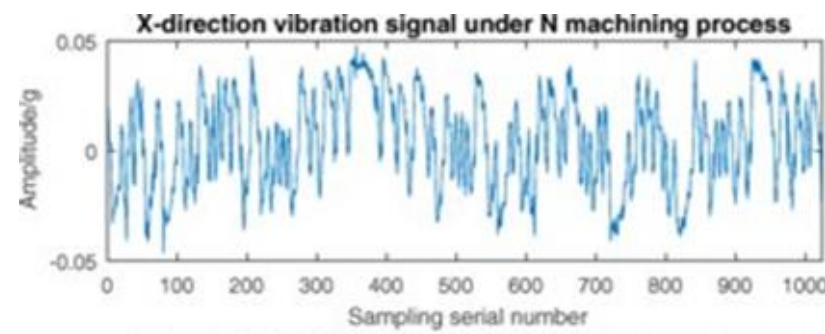

$X$-direction vibration signal under $T$ machining process

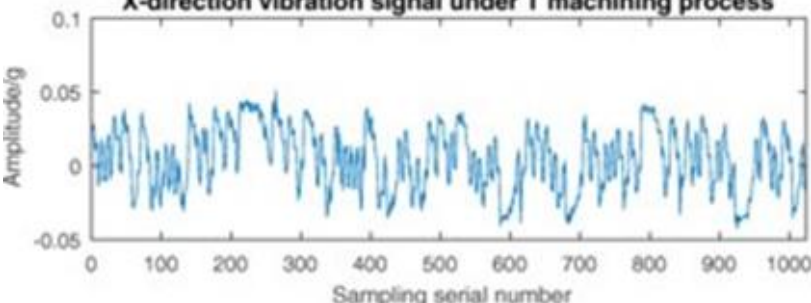

$\mathrm{X}$-direction vibration signal under $\mathrm{D}$ machining process
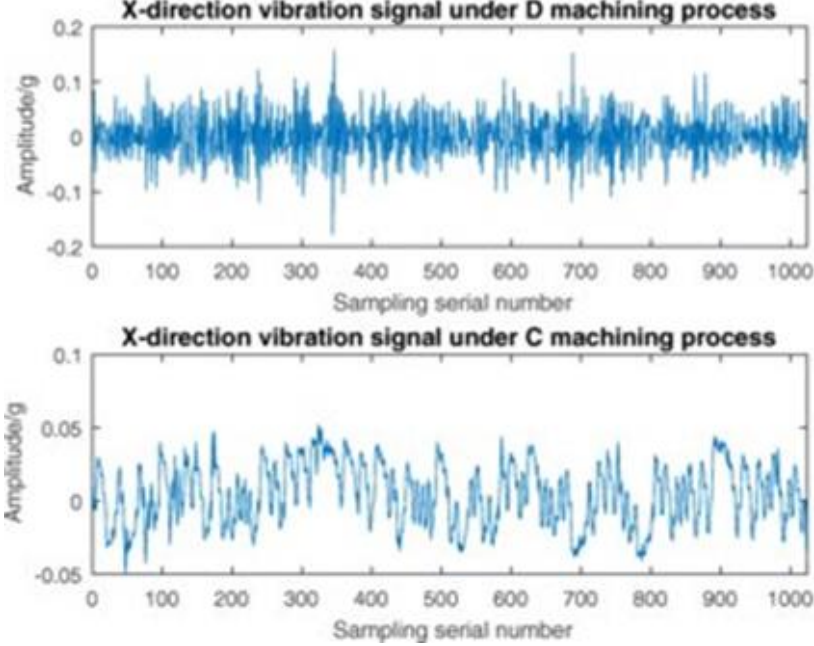

FFT of X-direction vibration signal under $\mathrm{N}$ machining process

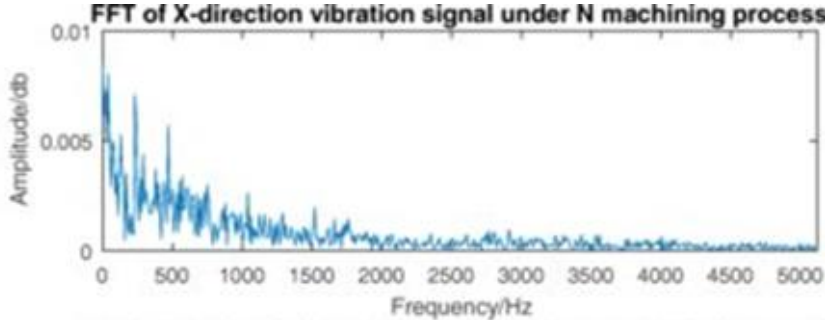

FFT of X-direction vibration signal under $\mathrm{T}$ machining process

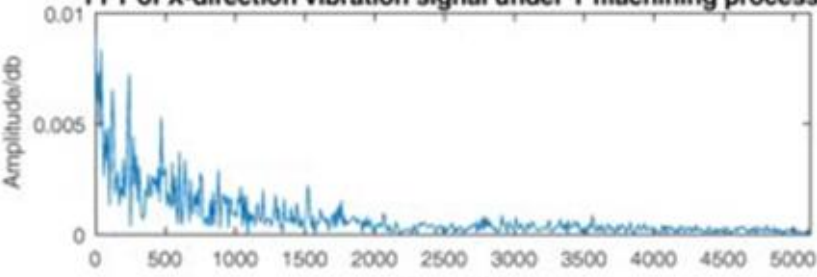

Frequency/Hz

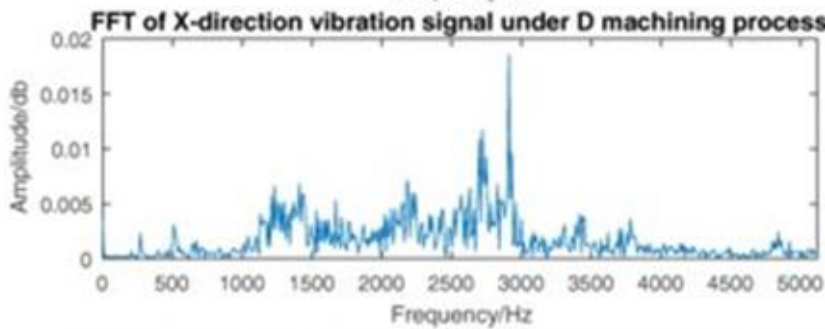

FFT of $\mathrm{X}$-direction vibration signal under $\mathrm{C}$ machining process

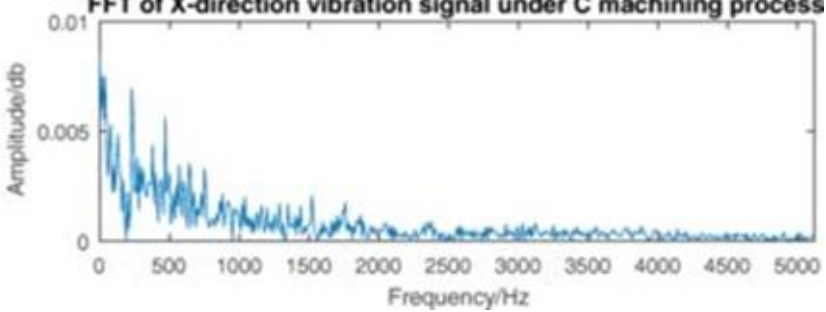

Figure 1. Vibration signal analysis in time domain and frequency domain under the single machining experiment condition

\section{Order Analysis}

Order analysis is a technique for analyzing noise and vibration signals in rotating or reciprocating machinery. Some examples of rotating or reciprocating machinery include aircraft and automotive engines, compressors, turbines, and pumps. Such machinery typically has a variety of mechanical parts such as a shaft, bearing, gearbox, blade, coupling, and belt. Each mechanical part generates unique noise and vibration patterns as the machine operates. Each mechanical part contributes a unique component to the overall machine noise and vibration. When performing vibration analysis many sound and vibration signal features are directly related to the running speed of a motor or machine such as imbalance, misalignment, gear mesh, and bearing defects. Order analysis is a type of analysis geared specifically towards the analysis of rotating machinery and how frequencies change as the rotational speed of the machine changes. It resamples raw signals from the time domain into the angular domain, aligning the signal with the angular position of the machine. This negates the effect of changing frequencies on the FFT algorithm, which normally cannot handle such phenomena.

\section{E. Time synchronous Averaging}

Time Synchronous Averaging (TSA) is a fundamentally different process than the usual spectrum averaging that is generally used in FFT analysis. While the concept is similar, TSA results in a time domain signal with lower noise than would result with a single sample. An FFT can then be computed from the averaged time signal. The signal is sampled using a trigger that is synchronized with the signal. The averaging process gradually eliminates random noise because the random noise is not coherent with the trigger. Only the signal that is synchronous and coherent with the trigger will persist in the averaged calculation. Traditional spectrum based averaging records a frame of data in the time domain, computes the FFT and then adds the FFT spectrum to the averaged spectrum. The time signal is discarded and then the process is repeated until the averaging number is complete. 


\section{International Advanced Research Journal in Science, Engineering and Technology}

Vol. 8, Issue 9, September 2021

DOI: $10.17148 /$ IARJSET.2021.8922

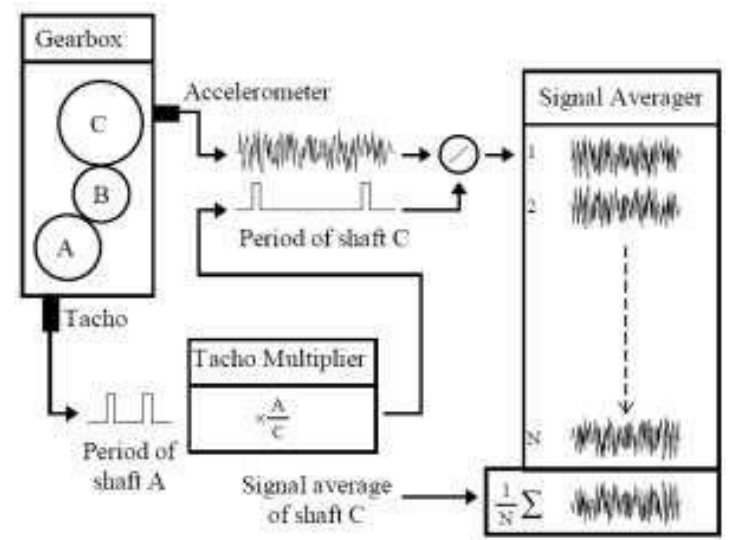

Figure 2. Synchronous signal averaging for time synchronous linear average

\section{OIL DEBRIS ANALYSIS}

Oil analysis is used extensively to help companies maintain their equipment. In order to take full benefit from the test data, it is important to understand the basic properties of a lubricant. Equally important is the understanding of how these properties affect the ability of the lubricant to function. Lastly, knowledge of the common test methods and instrumentation used to analyze oil will aid in data interpretation and lead to more productive corrective action. To effectively monitor how well a lubricant is functioning, you must first examine what the functions of the lubricant actually are. The primary function of a lubricant is quite obviously to lubricate. Lubrication can be defined as the reduction of friction. By reducing friction, wear is reduced, as is the amount of energy required to perform the work. Lubricants are also tasked with controlling the temperature of your equipment. Oil absorbs heat generated at the friction surface and carries it away to be dispersed. Many systems incorporate heat exchangers or radiators to aid in removing heat from the system. Along with heat, lubricants transport dirt and other debris away from the friction surface.

A.

Role of Oil Analysis

Selecting the proper lubricant, along with careful maintenance of that lubricant, is essential to ensure adequate protection to any machine. Proper lubrication is defined as a correct amount of the correct lubricant at the correct time. Maintaining lubricants means ensuring that the lubricant used in machine has the correct viscosity and have the necessary additives for the application. There are some steps to keep the lubricant clean and serviceable. Bottom line - oil analysis is the most effective way to prolong the useful life of your lubricants while maintaining maximum protection of your equipment. Oil analysis tests reveal information that can be broken down into three categories:

1) Lubricant condition:

Assessment of the lubricant condition reveals whether the system fluid is healthy and fit for further service, or is ready for a change.

\section{2) Contaminants:}

Increased contaminants from the surrounding environment in the form of dirt, water and process contamination are the leading cause of premature machine degradation and failure. Increased contamination alerts you to take action in order to save the oil and avoid unnecessary machine wear.

\section{3) Machine wear:}

An unhealthy machine generates wear particles at an exponential rate. The detection and analysis of these particles assist in making critical maintenance decisions. Machine failure due to worn out components can be avoided. Remember healthy and clean oil lead to the minimization of machine wear

Lubricant condition is monitored with tests that quantify the physical properties of the oil to ensure that it is serviceable. Metals and debris associated with component wear are measured to monitor equipment health. Lastly, some tests target specific contaminants that are commonly found in oils. It is imperative to select the proper blend of tests to monitor the machine's lubricant condition, wear debris and contaminants in order to meet the goals of successful oil analysis.

\section{B. Obtaining a representative sample}

Oil analysis is not merely a tool to analyze the condition of a lubricant. With modern diagnostic tools, it is used to monitor the condition of equipment as well. By utilizing these advanced techniques, equipment reliability can increase, and unexpected failures and downtime are minimized. There are many wear mechanisms that lead to the deterioration of machine components. Though there are different types of wear, there are only a few primary sources of wear.

The mechanisms that contribute to wear of a component include misalignment, unbalance and improper use of the equipment (such as overload or accelerated heating conditions). One of the sources for wear can be the lubricant itself 
e.g. in cases where the lubricant has degraded or has become contaminated. The different types of wear that can occur are:

- Abrasive wear

- Adhesive wear

- Cavitation 1

- Corrosive wear

- Cutting wear

- Fatigue wear

- Sliding wear.

In an operating machine, there is a continuous attrition of its components due to the generation of wear debris. Lubricants normally carry the debris away. Identification and analysis of this wear debris can pinpoint the type of wear and also identify the source, which could be any component under distress. Oil analysis can highlight the necessity to initiate a corrective action to prevent possible breakdowns. In this way, it becomes an important predictive maintenance technique. There have been cases where oil analysis helped to identify rotating equipment defects even before a vibration analysis could detect it. This especially applies to slow speed machines with higher load levels, like diesel engines. When implementing an oil analysis condition-monitoring program, it is important to select proper tests that will identify abnormal wear particles in the oil. The program should be customized to the type of equipment being monitored and the expected failures that are anticipated. The types of tests, location of sampling, analyses and the interpretation of oil analysis depend heavily on whether the application is a compressor, steam turbine, diesel engine, gearbox or a hydraulic system.

\section{Particle Behavior in a Machine}

The underlying reason that quantitative information can be used to signal a changing wear situation is that the particle concentration for normally operating equipment reaches a dynamic equilibrium. Experience has shown this to be true for diverse equipment. A mathematical model, given a few simplifying assumptions, has been constructed which demonstrates how equilibrium concentration is reached. This diagram of a hypothetical machine that, at given operating parameters such as load, speed, fluid inlet temperature, etc., generates X particles per unit volume of oil that passes through it. Here it is assumed that oil flow rate is constant.

The sampling valve is located immediately downstream of the machine so that if a cycle is begun with clean oil in the sump, the oil at the sampling valve for the first cycle of the oil through the machine (cycle here refers to the time for all the oil to pass through the system once) contains $\mathrm{X}$ particles per unit volume. Now, during passage of the oil through the system, there are various mechanisms for the removal of the particles. These are:

(1) filtration

(2) settling

(3) impaction and adhesion (sticking to solid surfaces)

(4) comminution1 (the grinding up of particles)

(5) dissolution (oxidation or other chemical attack)

(6) magnetic separation (as occurs in electric machinery)

For purposes of developing this model, it is assumed that these mechanisms are all proportional to the number of particles present which is reasonable if the particle dispersion is sufficiently dilute; that is, if particles do not interact with other particles to form agglomerates 1 or that particles are mutually repelled. Oil loss will not be considered in this model. Clearly, these removal mechanisms are a strong function of particle size, density, and shape. So, how long a machine should be operating before a representative sample is taken. For a machine with small oil capacity, high flow rate, and efficient filtration, the time will be short, whereas longer time is required for unfiltered systems with greater oil capacity and lower flow rate. If the concentration of small particles is of concern, greater time to equilibrium is predicted because removal mechanisms are less efficient for small particles.

\section{Elemental Spectroscopy}

Elemental spectroscopy is a test that has the distinction of monitoring all three categories - lubricant condition, wear debris and contaminants. A spectrometer is used to measure the levels of specific chemical elements present in an oil. Two types of spectrometers are commonly used. Arc emission spectrometers apply energy in the form of an electric arc to the sample. This excites the atoms into vapor form, creating a spectrum where light is generated. Individual light frequencies in the spectrum are measured and quantified to determine the presence and quantities of specific elements present. The other common type of spectrometer is the ICP (inductively coupled plasma) spectrometer. This operates on a similar principle, except that the energy is applied to the sample by a plasma flame rather than an electric arc. There are typically 20 elements measured by spectroscopy and reported in parts-per-million (ppm). These measurements represent elements in solution. Spectroscopy is not able to measure solid particles larger than roughly seven $\mu \mathrm{m}$, which leaves this test blind to larger solid particles. Typical levels of wear can vary greatly, depending on the type of equipment being sampled. For example, a gearbox will normally have much higher levels of iron than a hydraulic system. Levels of wear metals can vary across different units of the same type, depending on oil hours, operating conditions and loading 


\section{International Advanced Research Journal in Science, Engineering and Technology}

Vol. 8, Issue 9, September 2021

DOI: 10.17148/IARJSET.2021.8922

levels or other conditions. For this reason, it is impossible to establish firm limits for any piece of equipment based solely on the equipment type. To take full advantage of monitoring wear, a trend should be established to provide an poses a serious threat to the equipment. Water is a very poor lubricant and promotes rust and corrosion to the components. Dissolved water in oil will promote oil oxidation and reduce the load handling ability of the oil. Water in any form will cause accelerated wear, increased friction, and high operating temperatures. If left unchecked, water will lead to premature machine failure. In most systems, water should not exceed $500 \mathrm{ppm}$.

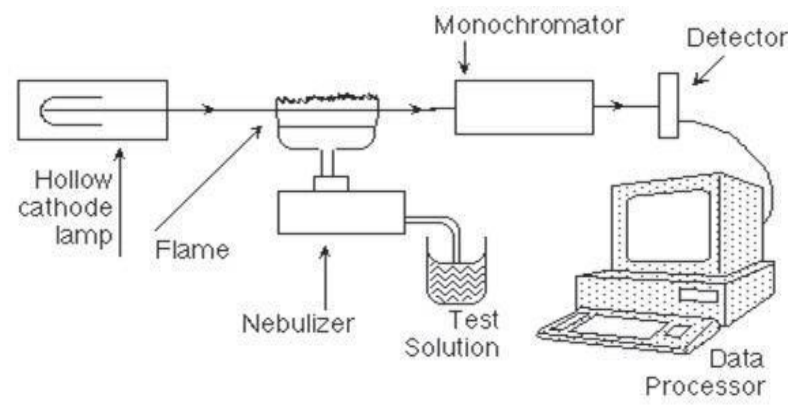

Figure 3. Block diagram of Elemental Spectroscopy

Engine oils will typically contain anti-wear additives composed of zinc and phosphorus. One should expect to see these elements present in approximately $1,000 \mathrm{ppm}( \pm 200 \mathrm{ppm})$. A detergent package should also be present, composed of some configuration of barium, magnesium and calcium. These levels will vary, depending on the oil.

Extreme pressure oils are typically for gear applications. It is common to see significant amounts of phosphorus.

Anti-wear oils include many bearing oils, some gear oils and hydraulic fluids. These oils contain both zinc and phosphorus from 200 to $600 \mathrm{ppm}$. There may be very low levels of detergent (magnesium or calcium) present also.

Rust and oxidation inhibiting oils are the easiest to identify. They include turbine oils, compressor oil, and some bearing and hydraulic oils. These oils have no metallic additives that can be measured by spectroscopy, so there should be extremely low numbers for all additive metals .It is not uncommon to see low levels $(<20 \mathrm{ppm})$ of some additive metals where they are not expected. This is usually the result of residual contamination in the equipment or storage tanks. There are oils that do not fit into these descriptions. Many oils are formulated for specific applications and alternative additives must be used. An example would be oils formulated for some stationary and electro motive diesel engines. In many cases, operating conditions or emission concerns call for a less traditional additive package.

E.

\section{Viscosity}

Viscosity is considered oil's most important property. The most common technique for measuring an oil's viscosity is following ASTM D445, using a viscometer (ASTM, 2011). A small sample of the oil is drawn into a calibrated capillary tube in a constant-temperature bath. Once the sample comes to temperature, it is allowed to flow down the tube a predetermined distance. The viscosity is the product of the flow time and tube calibration factor. The results are reported as the oil's kinematic viscosity in centistokes (cSt). When an oil's viscosity increases, it is usually due to oxidation, degradation or contamination. This is the result of extended oil drain intervals, high operating temperatures, or the presence of water or another oxidation catalyst. Increased viscosity can also be the result of excessive contamination with solids such as soot or dirt, as well as topping off with a higher-grade lube. Water contamination can also cause high viscosity A decrease in the oil's viscosity is most commonly due to contamination with fuel or a solvent. An oil's viscosity also can be affected if the wrong oil is used for top-off or replenishment. If a lubricant does not have the proper viscosity, it cannot properly perform its functions. If the viscosity is not correct for the load, the oil film cannot be established at the friction point. Heat and contamination are not carried away at the proper rates, and the oil cannot adequately protect the machine.

F.

\section{Acid Number}

Acid number (AN) is an indicator of oil health. It is useful in monitoring acid buildup in oils due to depletion of antioxidants. Oil oxidation causes acidic byproducts to form. High acid levels can indicate excessive oil oxidation or depletion of the oil additives and can lead to corrosion of the internal components. By monitoring the acid level, the oil can be changed before any damage occurs.

An oil analyst is looking for a sudden increase. When your oil is flagged for high acid levels, it indicates accelerated oil oxidation, and you should change the oil as soon as possible. If any of the remaining highly acidic oil is left, it will quickly deplete the antioxidants in the new oil. Acid number is measured by titration using ASTM D664 or D974. Both methods involve diluting the oil sample and adding incremental amounts of an alkaline solution until a neutral endpoint is achieved. The acid number of a new oil will vary, dependent upon the base oil additive package. An R\&O oil will usually have a very low AN around 0.03. An AW or EP oil will have a slightly higher value, typically around 0.5 . Engine oils commonly have a higher AN, in the neighborhood of 1.5 . 


\section{International Advanced Research Journal in Science, Engineering and Technology}

Vol. 8, Issue 9, September 2021

DOI: 10.17148/IARJSET.2021.8922

G.

Water Contamination

Water contamination is detrimental to any lubricant. A simple crackle test is used to determine if water is present in oil. A small volume of the lubricant is dropped onto a hot plate and, if bubbles or crackles occur, water is present. Low levels of water $(<0.5 \%)$ are typically the result of condensation. Higher levels can indicate a source of water ingress. Water can enter a system through seals, breathers, hatches and fill caps. Internal leaks from heat exchangers and water jackets are other potential sources. When free water is present in oil, it poses a serious threat to the equipment. Water is a very poor lubricant and promotes rust and corrosion to the components. Dissolved water in oil will promote oil oxidation and reduce the load handling ability of the oil. Water in any form will cause accelerated wear, increased friction, and high operating temperatures. If left unchecked, water will lead to premature machine failure. In most systems, water should not exceed 500 ppm.

H.

Particle Count

Particulate contamination has negative effects on all types of equipment. Particle count testing is a way to monitor the level of solid contamination in the oil. Two types of automatic particle counters are used to test oil cleanliness: light blockage and pore blockage.

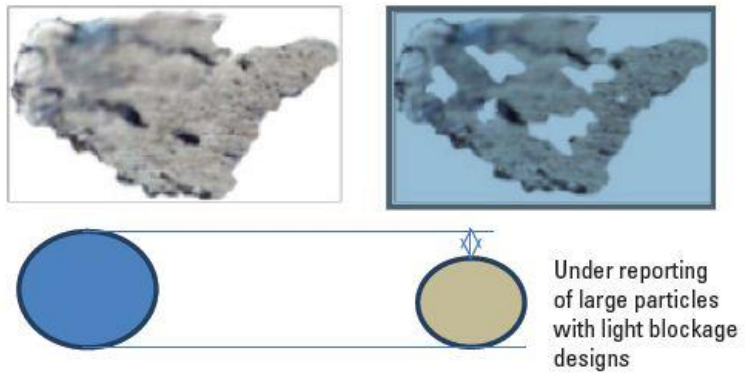

Figure 4 : Particle count in oil debris

I.

\section{Light blockage}

The light blockage technique involves passing a sample through a small orifice that has a laser light source on one side and an optical sensor on the other side. Particles interrupting the light beam are counted, and size is determined by the degree of light blockage. Light blockage particle counting is not effective when oil is contaminated with water or when air is entrained in the oil. In these circumstances, water or air bubbles will be counted as particles, causing erroneous results.

Pore blockage: The pore blockage or flow decay technique passes the sample through a mesh filter. As a filter clogs, the flow of the sample is slowed. The amount of flow decay is calculated, and the particle count can then be extrapolated. Because water droplets and entrained air will not restrict the fluid flow, there is no interference from these contaminants.

\section{J. Analytical Ferrography}

Analytical ferrography is used to separate solid contamination and wear debris from a lubricant for microscopic evaluation. As stated earlier, spectroscopy is not able to measure wear particles larger than $7 \mu \mathrm{m}$ in size. While particle counting, ferrous wear concentration and DR ferrography are able to detect the presence of larger particles, they cannot qualify their composition or

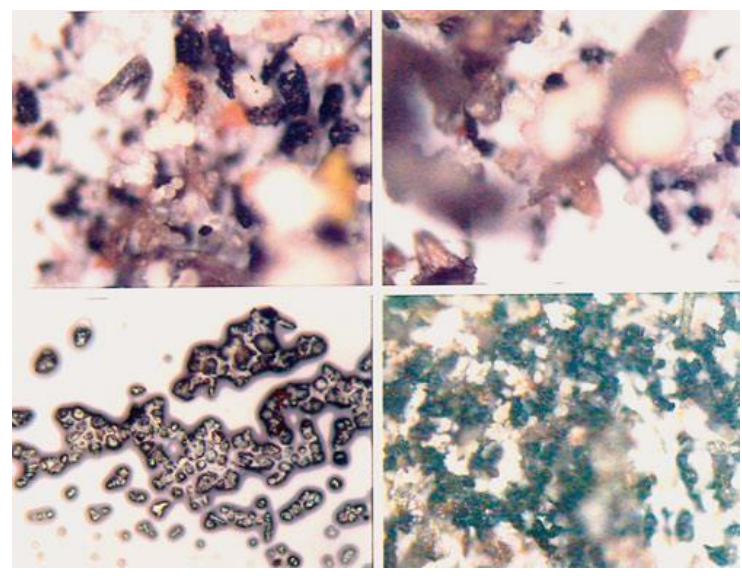

Figure 5 : Analytical Ferrography 


\section{International Advanced Research Journal in Science, Engineering and Technology}

Vol. 8, Issue 9, September 2021

DOI: 10.17148/IARJSET.2021.8922

origin. Analytical ferrography is able to identify wear particles, their composition and their origin by visually analyzing them microscopically (Fig. 5). A diluted oil sample is allowed to flow over a specially treated slide positioned at an angle over a strong magnet. The ferrous (iron) particles are attracted to the magnet and deposited onto the slide in decreasing size as the oil flows down the substrate. Nonferrous particles are deposited randomly, while ferrous particles line up in chains as a result of the magnetic flux. The result is a microscopic slide with the particles separated by size and composition. Microscopic examination of the debris reveals information about the condition of the equipment. Observing the concentration, size, shape, composition and condition of the particles indicates where and how they were generated. Particles are categorized based on these characteristics, and conclusions can be drawn regarding the wear rate and health of the machine.

The composition of the particles can be identified by color. Heat treating the slide causes specific color changes to occur in various types of metals and alloys. The particle's composition indicates its source. The particle's shape reveals how it was generated. Abrasion, adhesion, fatigue, sliding and rolling contact wear modes each generate a characteristic particle type in terms of its shape and surface condition. Solid contaminants can also be visually identified, provided they are of a commonly found origin. Sand and dirt, fibers, oxidation products, rust and metal oxides are examples of contamination debris that can be identified.

\section{CONCLUSION}

The Fault diagnosis and condition monitoring system in very important for a gear system as it has to carry heavy load and work in extreme condition. To ensure its performance and minimize damaging workflow a gear box should be monitored regularly. Vibration monitoring and oil analysis both are effective to monitor the condition. We can also use integrated system with both methods together to gain more accurate data about a failure before it occurs.

\section{REFERENCES}

1. Oil Analysis Overview; School of mechatronic engineering, China university of mining and technology.

2. Aherwar, Amit \& Khalid, S. (2012). Vibration analysis techniques for gearbox diagnostic: A review. Int. J. Adv. Eng. Technol. 3. 4-12.

3. Prediction on wear of a spur gearbox by on-line wear debris concentration monitoring; Song Feng, Bin Fan, Junhong Mao, Youbai Xiea.

4. Gear Damage Detection Using Oil Debris Analysis; Dempsey, Paula J. (NASA Glenn Research Center, Cleveland, OH United States)

5. analysis to monitor the performances of three $90 \mathrm{~kW}$ gearboxes; E.D.Yardley

6. Root cause and vibration signal analysis for gearbox condition monitoring; Bartelmus

7. The combined use of vibration, acoustic emission and oil debris on-line monitoring towards a more effective condition

8. monitoring of rotating machinery; T.H.Loutas, D.Roulias, E.Pauly, V.Kostopoulos

9. A robust diagnostic model for gearboxes subject to vibration monitoring; Yimin Zhan, Viliam Makis

10. USINGVIBRATIONMONITORING FOR LOCAL FAULT DETECTIONONGEARSOPERATING UNDER

11. FLUCTUATING LOADCONDITIONS; C. J. Stander and P. S. Heyns

12. Understanding Oil Analysis: How It Can Improve Reliability of Wind Turbine Gearboxes; Michael P. Barrett and Justin

13. Monitoring wind turbine gearboxes; Yanhui Feng1, Yingning Qiu1, Christopher J. Crabtree2, Hui Long3 and Peter J. Tavner

14. Investigation of Various Condition Monitoring Techniques Based on a Damaged Wind Turbine Gearbox; S. Sheng

15. https://www.machinerylubrication.com/Read/30111/analytical-ferrography-value

16. https://www.researchgate.net/publication/302931893_Vibration_analysis_techniques_for_gearbox_diagnostic_A_review

17. https://ieltsonlinetests.com/zh-hans/ielts-exam-library\#academic

\section{BIOGRAPHY}

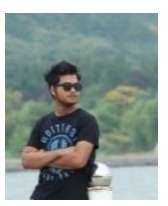

\section{S M NAUFEL ISALM}

S M Naufel Islam is a undergraduate student at school of mechatronics engineering in China university of mining and techonology.He will be graduate in june 2022. His research interests include Nondestructive testing , Advanced manufacturing, Robotics.

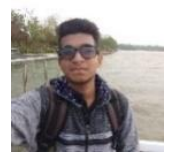

\section{MD farhan Mahmud Rashik}

From the very beginning Farhan Mahmud Rashik had a strong interest in engineering studies which resulted him going abroard to study mechanical engineering. He is a undergrad student of CUMT. He has completed a project in robotics programming at the China University of Mining Technology Conference. In the future he is willing to work on any research topics, Specifically in mining engineering and mechanical engineering.

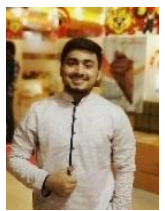

\section{MD Khairul Basher}

MD Khairul Basher is a mechanical engineering student from china university of mining and technology.He has compleated his three years successfuly as a international student with a lot acadamic achivment.He is intrested in various research field of engineering.He wants to be a automation engineeer in future. 